\title{
Workflow Deployment in ICENI II
}

\author{
A. Stephen McGough, William Lee, and John Darlington \\ London e-Science Centre \\ Department of Computing \\ Imperial College London, London \\ \{asm, wwhl, jd\}@doc.ic.ac.uk
}

\begin{abstract}
The Imperial College e-Science Networked Infrastructure (ICENI) has been developed by the London e-Science Centre for over four years. ICENI has prototyped many novel ideas for providing an end to end Grid middleware. This has included: service-oriented architecture, component programming model, retaining and using meta-data collected throughout the life-cycle of an application, and scheduling algorithms which are aware of workflow and performance data. In this paper we describe the workflow pipeline and deployment process of ICENI II. This allows the user to specify their workflow at an abstract level which is fed through the pipeline in order to successfully deploy it over the Grid. The tool sets, which make up the stages of the ICENI II pipeline, are designed to be composable in an à-la-carte fashion. Thus allowing Grid developers to select only those components which are relevant for their work. When these tool sets are composed together they form the higher level services required to make the Grid useful to the end user.
\end{abstract}

\section{Introduction}

The ICENI (Imperial College e-Science Networked Infrastructure) [8, 3] has been developed as a service-oriented architecture for (workflow) component composition. Our focus in ICENI has three major elements: prototyping the services and their interfaces necessary to build a service-oriented middleware; developing an augmented component programming model for authoring reusable Grid applications; to explore the meta-data needed to enable effective machine-assisted and automated decision-making; and the development of higher level services required to make Grid infrastructure usable. ICENI II is a re-working of the original ICENI concepts using the newly evolving commodity tools, standards and services. Allowing us to re-focus on the higher level services required to make the Grid useful to the scientist. In this paper we present the workflow pipeline and deployment service for ICENI II focusing on the core services that can be composed to enable a (workflow) component programming paradigm.

In ICENI an application is a composition of components and services defined using a workflow language. A component is an indivisible unit of execution in which all the contextual, functional and behavioral aspects are made explicit. While multiple components might exhibit the same functional interface, they can be independently implemented yielding different behavior [10]. Furthermore, a 
component is an abstract entity that awaits composition by application authors and subsequent deployment into an execution environment.

A deployed component is referred to as a service. A service is a realised manifestation of the states and functions provided by the underlying resource and the component implementation. The definition does not mandate a particular component implementation architecture. The current ICENI II implementation requires the use of an interoperable set of protocols (e.g. SOAP) and data model (e.g. WSDL, XSD Schema) as the common denominator. We envisage the protocol details are sufficiently abstract from the component and application authors. This allows applications to be composed with both abstract components that need to be provisioned and anchored services that exist irrespective of the lifetime of the application. It is the role of the workflow pipeline to realise an application by performing component implementation selection, resource allocation, deployment and orchestration. Further information on the ICENI component model can be found in [8, 9 .

\section{Architecture}

Figure 1 illustrates the workflow pipeline for ICENI II. Within this design we have determined three main stages within an application's life-cycle:

Specification of the application to be executed. At this stage the workflow may be abstract in nature - the code that will be used to implement the tasks and the

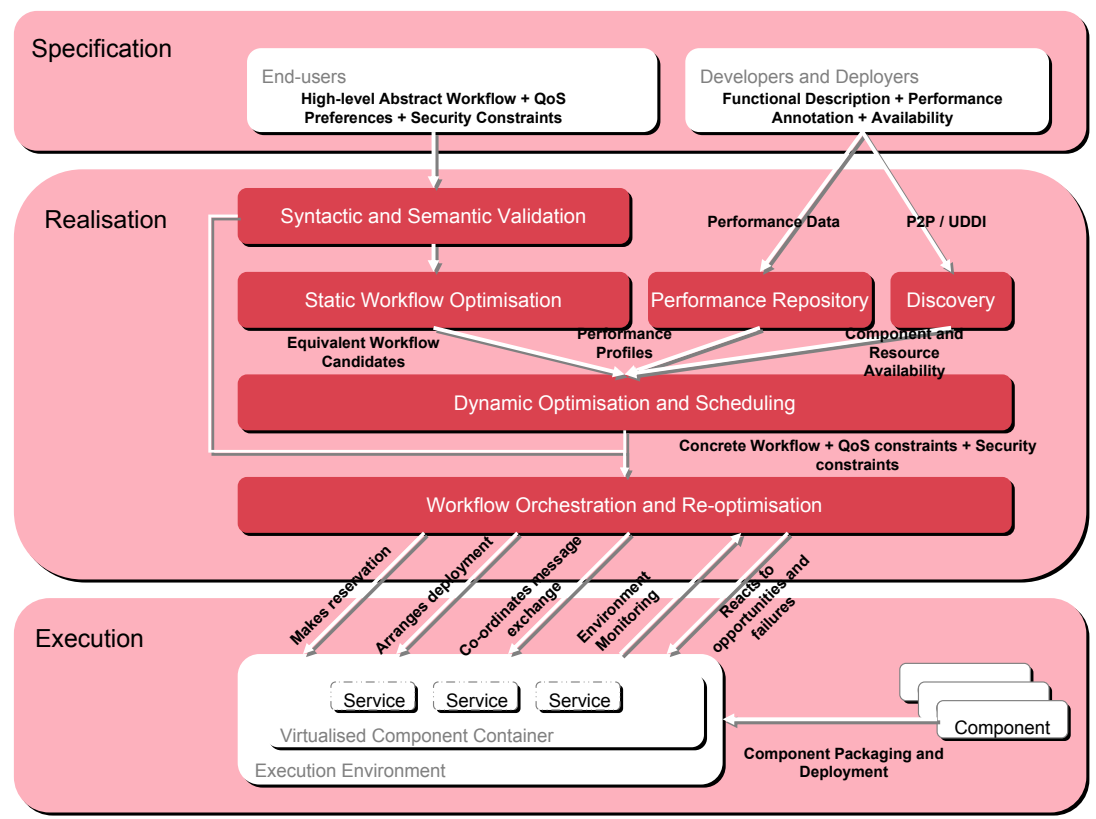

Fig. 1. The Design Diagram for ICENI II 
resources on which they run are not yet defined. The translation of an application scientist's ideas into these workflows is a complex task. It cannot be assumed that the application scientist will be an expert with workflow languages, we therefore see the need to develop application specific interfaces which allow the scientist to describe their problem in their own space - translating this down into a workflow. This is an area of active research. We are working with the bio-informatics community within the e-Protein project [13. The Taverna project [21] is a good example of this process.

Realisation of the workflow. The workflow needs to be made concrete before it can be executed. This process involves determining the appropriate resources and software (component implementations) dependencies for the workflow to operate. The Realisation stage of the life-cycle is discussed further in Section 3 , Execution of the workflow. In this stage the concrete workflow is enacted on the defined set of resources. Implementations will be made active on resources at the appropriate point in time. If however the workflow does not proceed as planned then the realisation stage can be re-entered so that the workflow may be altered in light of the changes. It is also possible to re-enter the realisation stage in cases where parts of the original workflow couldn't be made concrete at the original pass or when new opportunities become available; such as availability of new resources. Further details of the Execution stage are given in Section 4.

Once a workflow enters the realisation stage an Application Service will be designated to represent the running application. It acts as a central point where the user can find out about the state or influence the progress of the application.

\section{Realisation of Workflows}

The Realisation stage selects the best use of anchored services or resources and component implementations available on the Grid in an attempt to match the criteria specified by the application scientist. Most often this will be in terms of time constraints that the scientist may have. Although, other constraints may be used (or combined) such as cost of resources, reliability of execution or the level of trust required from the used resources and components. This is often referred to as a Quality of Service (QoS) Document. This stage will take the abstract workflow and resolve it into a concrete workflow in which implementations and resources will have been determined.

The Realisation stage can be decomposed into several complementary services with most being optional. The only non-optional element, referred to as the Resolver, matches abstract components with anchored services or implementations and resources. Pluggable optimisers can be used to improve the performance of the workflows or matching of QoS requirements. Detailed in the following subsections are the pluggable elements that we have defined so far [1].

Syntactic and Semantic validation. On entering the Realisation stage the workflow is checked for syntactic and semantic validity. The first step in checking syntactic correctness ensures that all required inter-component connections have 
been made, i.e. all ports that must be connected are, otherwise the workflow will be unsuccessful at a later stage. The second step is to verify that the data to be exchanged through each of these connections is a valid transaction.

The workflow may now be checked for semantic validation. Scientific knowledge about what action a component performs, and what the meaning of its inputs and outputs, can be used to determine if the connection of two components makes sense. For example it may be syntactically correct to feed a matrix into a finite difference solver, though if this matrix is a diagonal solution to a set of linear equations this makes little semantic sense. If a workflow fails at a syntactic or semantic level then it is returned to the specification stage.

Static Workflow Optimisation. The Workflow Static Optimisation Service is responsible for manipulation of the workflow. Using static information about the components and their composition this service generates a new workflow which is expected to execute more efficiently than the original. This is achieved through such steps as re-ordering of the components to improve efficiency; inserting additional components to allow particular implementations to communicate with each other; substitution of semantically equivalent workflow sections; pruning of redundant components from composed workflows; and the substitution of alternative implementations of components. It should be noted that the Workflow Static Optimisation Service does not consider the dynamic load on system's within the Grid.

Pruning of Resource Space. Once the workflow has been optimised it is now essential to reduce the set of resources that will be considered. This is achieved by looking at the slowly changing resource information such as authorization to use a resource; hardware / software requirements; problem specific requirements such as long execution time with no option to checkpoint; and sensibility of selection.

Workflow-aware, performance-guided scheduling. The aim of most schedulers is to map the abstract workflow to a combination of resources and implementations that is both efficient in terms of execution time of the workflow and in terms of the time to generate the concrete workflow. Components need not all be deployed at the same time: just in time scheduling of components and the use of advanced reservations help to make more optimal use of the available resources for both the current and other users.

Schedulers need to be designed to be workflow aware. A number of workflow aware schedulers have been prototyped in ICENI [12] and we are now developing new workflow aware performance guided schedulers using constraint equations solved using Mixed Integer Linear Programming [15]. Thus the scheduling of components depends not only on the performance of a component on a given resource, but also on the effect this will have on the other components in the workflow. Described below are the general steps taken to evaluate a suitable mapping of components onto resources.

The scheduler can speculatively match implementations with resources. The scheduler can then interrogate performance information in order to obtain estimates on the execution times for these implementation / resource combinations 
along with any implications for the overall workflow. With this information and information gathered from the resources that have been discovered, the scheduler can determine an appropriate mapping of the components over the resources.

\section{Execution of a Workflow}

Due to the uncertainties of resource and network availability in a dynamic system such as the Grid, it is necessary to support advanced reservations to provide QoS guarantees. Reservations may be made on computational resources, storage resources, instruments or the underlying fabric of the Internet such as network links. The reservations may be made for exclusive use of the entity or, in some cases, some pre-agreed portion of it. This can be realised through the introduction of market forces into the Grid [6].

The execution environment represents the virtualisation of the resource that manages the life-cycle of the parts of an application (see Figure 21). The execution environment encapsulates the facilities available to the software component, such as inter-component communication, logging, monitoring, failure recovery, checkpointing and migration. These facilities are exposed to the software component through a set of abstract APIs. These abstractions allow the execution environments managing the parts of an application to co-operate and co-ordinate their runtime capabilities, such as network transport, co-location and shared file system. Software engineers developing the components are insulated from the implementation choice made by the optimisation stage by following the software patterns offered by the APIs. This is analogous to the MPI[5] abstraction for message-passing in parallel applications.

The software component instantiated in the execution environment is referred to as a service. We adopt Web Services as one view of the running software component. It is an ideal way for services on different physical resources to communicate with each other in an interoperable manner. The elements in the execution environment will be discussed in more detail.

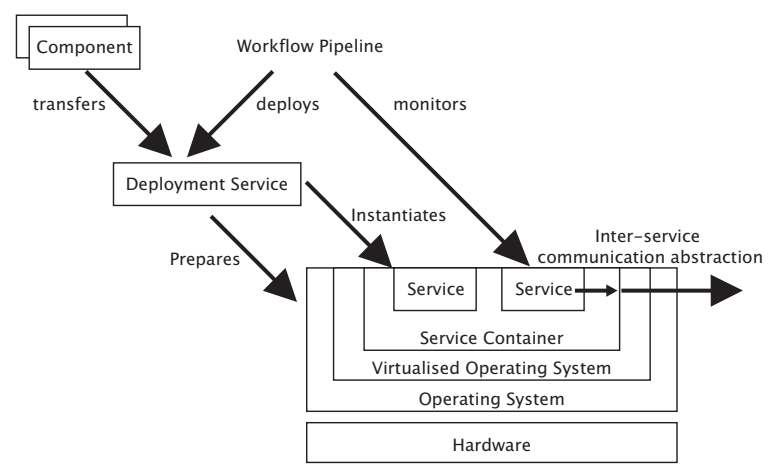

Fig. 2. Execution Environment and Multi-level Virtualisation 


\subsection{Component Deployment}

A deployment service is the gateway to a computational resource. It is responsible for facilitating the provisioning and instantiation of a component assigned to a particular resource. Firstly, the deployment service prepares the execution environment. This might involve the preparation of a component container in a cluster resource. Recent advances in virtualisation technologies 1, 20, offer operating system-level virtualisation. Within the virtualised operating system, a component container provides the higher-level abstraction to the software component on top of the operating system facilities. The compartment model offers attractive features such as security and fault isolation. Multi-level virtualisation allows runtime facilities to be flexibly configured depending on the deployment requests [16]. Although virtualisation provides a sandbox environment for a component to execute seemingly exclusively, the cost in instantiating the container on-demand [7] may be too high for short-running components. Predictive instantiation might alleviate the setup cost by allocating resources in advance.

Once an execution environment is available, the deployment service will facilitate the provision of the software component onto the resource. This might involve the staging of software packages and their dependencies available remotely into the system. In order for this architecture to succeed across the Grid, a standardised interface for deployment and a language for software requirement description is essential. It reduces the need for users and software agents to understand a large number of description languages and deployment mechanisms to exploit a variety of Grid resources.

GridSAM [4] is a job submission and monitoring Web Service developed through collaboration with the OMII 14. GridSAM allows the deployment of legacy code through a standard interface and is seen as one of the first toolkit items within ICENI II.

\subsection{Checkpointing and Migration}

Checkpointing is a technique for preserving the state of a process in order to reconstruct it at a later date. It is a crucial element for providing fault-recovery from a saved state. In scientific applications checkpointing provides a means for long-running simulations to be restarted at a previously examined parameter space [2]. This is also an important means for migrating the state of a process to another execution environment. This is often triggered by a re-scheduling decision as in some distributed resource managers. Many checkpointing and migration systems exist including OpenMosix [18], OpenSSI [19], and Kerrighed [17].

Migration might occur as a result of a recovery operation after a host failure. Alternatively migration may be as the result of a desire to exploit new possibilities within the Grid, such as a new resource becoming available.

It is worth pointing out that not all components can be checkpointed. Techniques to checkpoint components are outlined below. The generality of the approaches increases from one to the next. Checkpointing and migration schemes can be classified into three broad categories. Application-level checkpointing 
might be initiated by the application itself through a checkpointing and migration API. This provides fine-grain control to the developer to save the states of the application at a critical moment of the execution, however it requires existing applications to be modified to take advantage of the functionality. Existing executables could also be made checkpointable by linking to checkpoint libraries that capture running stack, heap and program counter information in order to reconstruct the process remotely. This solution produces a checkpoint image that is rarely portable and complete because network sockets or file handles are inherently difficult to reconstruct. System level checkpointing provided by many virtual machine technologies allows the whole virtualised environment to be checkpointed. This provides a generic solution for most cases but the coarse nature means the checkpoint image would be very large.

In cases where the services within the workflow are capable of being checkpointed and migrated, the application co-ordinator in the workflow pipeline may re-schedule the service state to a suitable resource in order to respect the stated quality constraints. This might involve re-scheduling other services to different resources to achieve an optimal schedule. The stored checkpoints of services are transferred to the suitable resources through the deployment service and restarted in a reinstated execution environment. A component might receive messages during the time elapsed between its failure and restart. Such events are taken care of by the messaging abstraction in the initiating execution environment. The execution environment reports any anomalies like resource overloading or network failure to the application co-ordinator which in turn might trigger the migration process.

\section{Conclusion}

In this paper we have outlined a set of high level tool sets for the manipulation of workflows. When composed together these tool sets form the stages of a workflow pipeline which take a users abstract workflow through to deployment on Grid resources.

We are now developing some of these higher level services on top of GridSAM for job deployment and monitoring. Along with developments in the other services for optimising workflows across multiple resources.

Wherever possible, and appropriate, ICENI II is being developed using existing standards. To this end ICENI II will be developed as a set of Web Services and using up and coming standards such as BPEL4WS and JSDL.

\section{References}

1. P. Barman, B. Dragovic, K. Fraser, S. Hand, T. Harris, A. Ho, R. Neugebauer, I. Pratt, and A. Warfield. Xen and the art of virtualization. In SOSP 2003, September 2003.

2. J. Chin, J. Harting, S. Jha, P.V. Coveney, A. R. Porter, and S. M. Pickles. Steering in computational science: mesoscale modelling and simulation. Contemporary Physics, 44:417-434, 2003. 
3. N. Furmento, A. Mayer, S. McGough, S. Newhouse, T. Field, and J. Darlington. ICENI: Optimisation of Component Applications within a Grid Environment. Journal of Parallel Computing, 28(12):1753-1772, 2002.

4. Grid Submission and Monitoring service (GridSAM). http://www.lesc.imperial.ac.uk/gridsam

5. W. Gropp, E. Lusk, N. Doss, and A. Skjellum. A high-performance, portable implementation of the MPI message passing interface standard. Parallel Computing, 22(6):789-828, September 1996.

6. Jeremy Cohen and John Darlington and William Lee. Payment and Negotiation for the Next Generation Grid and Web. In UK e-Science All Hands Meeting, Nottingham, UK, sep 2005.

7. K. Keahey, K. Doering, and I. Foster. From Sandbox to Playground: Dynamic Virtual Environments in the Grid. In 5th IEEE/ACM International Workshop on Grid Computing, November 2004.

8. A. Mayer, S. McGough, N. Furmento, J. Cohen, M. Gulamali, L. Young, A. Afzal, S. Newhouse, and J. Darlington. Component Models and Systems for Grid Applications, volume 1 of CoreGRID series, chapter ICENI: An Integrated Grid Middleware to Support e-Science, pages 109-124. Springer, June 2004.

9. A. Mayer, S. McGough, N. Furmento, W. Lee, S. Newhouse, and J. Darlington. ICENI Dataflow and Workflow: Composition and Scheduling in Space and Time. In UK e-Science All Hands Meeting, pages 627-634, Nottingham, UK, September 2003. ISBN 1-904425-11-9.

10. A. Mayer, S. McGough, M. Gulamali, L. Young, J. Stanton, S. Newhouse, and J. Darlington. Meaning and Behaviour in Grid Oriented Components. In 3rd International Workshop on Grid Computing, Grid 2002, volume 2536 of Lecture Notes in Computer Science, Baltimore, USA, November 2002.

11. A.Stephen McGough, Jeremy Cohen, John Darlington, Eleftheria Katsiri, William Lee, Sofia Panagiotidi, and Yash Patel. An End-to-end Workflow Pipeline for Large-scale Grid Computing. Journal of Grid Computing, pages 1-23, February 2006 .

12. S. McGough, L. Young, A. Afzal, S. Newhouse, and J. Darlington. Workflow Enactment in ICENI. In UK e-Science All Hands Meeting, pages 894-900, Nottingham, UK, sep 2004.

13. A. O'Brien, S.J. Newhouse, and J. Darlington. Mapping of scientific workflow within the e-protein project to distributed resources. In Proceedings of the UK e-Science All Hands Meeting 2004, Nottingham, September 2004.

14. Open Middleware Infrastructure Institute (OMII). http://www.omii.ac.uk/

15. Yash Patel, A. Stephen McGough, and John Darlington.

16. E. Smith and P. Anderson. Dynamic Reconfiguration for Grid Fabrics. In 5th IEEE/ACM International Workshop on Grid Computing, November 2004.

17. The Kerrighed project. http://www.kerrighed.org/

18. The open Mosix project. http://openmosix.sourceforge.net/

19. The open SSI project. http://openssi.org/index.shtml.

20. User Mode Linux. http://user-mode-linux.sourceforge.net/

21. The Taverna Project Website. http://taverna.sourceforge.net/ 